\title{
Hiperplasia Congénita da Suprarrenal por Deficiência de 21-Hidroxílase: Correlação Genótipo-Fenótipo
}

\author{
Congenital Adrenal Hyperplasia Due to 21-Hydroxylase Deficiency: \\ Genotype-Phenotype Correlation
}

Catarina MENDES ${ }^{1}$, Inês VAZ MATOS ${ }^{1}$, Luís RIBEIRO'1, Maria João OLIVEIRA ${ }^{1}$, Helena CARDOSO², Teresa BORGES ${ }^{1}$ Acta Med Port 2015 Jan-Feb;28(1):56-62

RESUMO

Introdução: A hiperplasia congénita da suprarrenal por deficiência de 21-hidroxílase constitui uma das doenças hereditárias mais comuns. Resulta de diferentes mutações no gene CYP21A2 e, na maioria dos casos, a gravidade da doença correlaciona-se com a variação alélica do CYP21A2. O objetivo deste estudo foi descrever o espectro mutacional do CYP21A2 e avaliar a correlação genótipo-fenótipo numa coorte de doentes portugueses com deficiência de 21-hidroxílase.

Material e Métodos: Estudo retrospetivo de 22 doentes com diagnóstico clínico de deficiência de 21-hidroxílase. Foi feita análise molecular do CYP21A2 e estabelecida a correlação genótipo-fenótipo.

Resultados: Foi realizada genotipagem em 22 doentes não relacionados: 5 com a forma clássica perdedora de sal (idade média ao diagnóstico de 10,2 dias; mínimo 1, máximo 20 dias), 7 com a forma clássica virilizante simples (idade média ao diagnóstico de 3,5 anos; mínimo 0 dias, máximo 7 anos) e 10 com a forma não clássica (idade média ao diagnóstico de 5,7 anos; mínimo 4 anos, máximo 8 anos). Os defeitos genéticos mais frequentes nas formas clássicas foram o I2 splice (24\%) e I172N (24\%), seguindo-se o Q318X $(16 \%)$ e deleções de genes (16\%) e, na forma não clássica, o V281L (80\%). Verificou-se uma concordância genótipo-fenótipo global de $81,8 \%$. O genótipo permitiu prever adequadamente o fenótipo em $83,3 \%, 100 \%$ e $90 \%$ dos doentes com mutações compatíveis com a forma clássica perdedora de sal, clássica virilizante simples e não clássica, respectivamente.

Discussão: A frequência de defeitos genéticos observados nos nossos doentes é comparável a estudos semelhantes. Observou-se, na maioria dos casos, uma boa correlação genótipo-fenótipo.

Conclusões: A análise molecular do CYP21A2 fornece informação importante relativamente à gravidade da doença e no aconselhamento genético e pré-natal.

Palavras-chave: Esteróide 21-Hidroxílase; Fenótipo; Genótipo; Hiperplasia Congénita Suprarrenal.

\section{ABSTRACT}

Introduction: Congenital adrenal hyperplasia due to 21-hydroxylase deficiency is one of the most frequent inborn conditions. It is caused by distinct mutations in the CYP21A2 gene and in the majority of cases the disease's severity correlates with CYP21A2 allelic variation Our aim was to describe the mutational spectrum of CYP21A2 and evaluate genotype-phenotype correlation in a cohort of portuguese patients with 21-hydroxylase deficiency.

Material and Methods: Retrospective study of 22 patients with clinical diagnosis of 21-hydroxylase deficiency. Molecular analysis of CYP21A2 was performed and genotype-phenotype correlation was then established.

Results: Genotyping was performed in 22 unrelated patients: 5 with classic salt-wasting (average age of diagnosis 10.2 days; minimum 1 , maximum 20 days), 7 with classic simple virilizing (average age of diagnosis 3.5 years; minimum 0 days, maximum 7 years) and 10 with nonclassical form (average age of diagnosis 5.7 years; minimum 4 years, maximum 8 years). The most frequent genetic defects in the classic forms were I2 splice (24\%) and I172N (24\%), followed by Q318X (16\%) and gene deletions (16\%) and in the nonclassical form, the V281L (80\%). The overall concordance between genotype and phenotype was $81,8 \%$. Genotype accurately predicted phenotype in $83.3 \%, 100 \%$ and $90 \%$ of patients with classic salt-wasting, classic simple virilizing and nonclassical mutations, respectively.

Discussion: The frequency of genetic defects in our patients was comparable to similar studies. In most cases there was a good correlation between genotype and phenotype.

Conclusions: Molecular analysis of CYP21A2 provides useful information in terms of prediction of disease severity, genetic and prenatal counseling.

Keywords: Adrenal Hyperplasia, Congenital; Genotype; Phenotype; Steroid 21-Hydroxylase.

\section{INTRODUÇÃO}

A hiperplasia congénita da suprarenal (HCSR) compreende um grupo de doenças hereditárias autosómicas recessivas que comprometem a síntese do cortisol. Trata-se de uma das patologias hereditárias mais comuns e cerca de $95 \%$ dos casos ocorrem por deficiência da enzima 21-hidroxílase (21-OH). ${ }^{1}$ Esta enzima é responsável pela conversão da 17-hidroxiprogesterona (17-OHP) em 11-desoxicortisol, um precursor do cortisol, e da progesterona em desoxicortisona, um percursor da aldosterona. ${ }^{2}$

O gene que codifica a 21-OH, o CYP21A2, localiza-se no braço curto do cromossoma 6 , em estreita proximidade ao seu pseudogene e altamente homólogo, o CYP21A1P. ${ }^{3}$ As mutações responsáveis pela deficiência de $21-\mathrm{OH}$ resultam, tipicamente, da recombinação desigual entre o CYP21A2 e o CYP21AP1, designadas conversão de genes. ${ }^{4} \mathrm{~A}$ maioria das mutações devem-se à transferência

1. Departamento da Criança e do Adolescente. Unidade de Endocrinologia Pediátrica. Centro Hospitalar do Porto. Porto. Portugal.

2. Serviço de Endocrinologia, Diabetes e Metabolismo. Centro Hospitalar do Porto. Porto. Portugal.

Recebido: 10 de Junho de 2014 - Aceite: 13 de Outubro de 2014 | Copyright $\odot$ Ordem dos Médicos 2015 
de sequências curtas do pseudogene para o CYPA21A2 durante a meiose. ${ }^{5}$ Cerca de $75 \%$ dos doentes são heterozigóticos compostos para as mutações responsáveis pela doença e a gravidade da doença é determinada pela atividade do alelo que se encontra menos gravemente afectado e, consequentemente, com a atividade residual da $21-\mathrm{OH} .^{6-9}$

A HCSR classifica-se em dois grupos: forma clássica e forma não clássica. A forma clássica é subdivida em forma clássica perdedora de sal (CPS) e forma clássica não perdedora de sal, também designada virilizante simples (CVS).

A forma clássica ocorre em cerca de 1 em cada 700015000 nascimentos, na maioria das populações. ${ }^{10} \mathrm{~A}$ incidência da forma não clássica encontra-se menos definida, mas estima-se que seja aproxidamente 1 em cada 1000 nascimentos. ${ }^{11}$

O que distingue a forma CPS da forma CVS é que, na primeira, os doentes são incapazes de sintetizar aldosterona suficiente para manter o balanço de sódio. ${ }^{6-9}$ Corresponde à forma mais grave da doença e afecta cerca de $75 \%$ dos indivíduos que manifestam a forma clássica. ${ }^{12}$

Para além dos sinais de virilização e hipocortisolismo, os doentes apresentam insuficiência mineralocorticóide, com predisposição para episódios de hipotensão, desidratação hiponatrémica com hipercalémia e choque hipovolémico potencialmente fatais no período neonatal. ${ }^{6-9,12}$ No sexo feminino, o diagnóstico é habitualmente precoce pela presença virilização genital, secundária ao excesso de androgénios durante a gestação. Nos recém-nascidos do sexo masculino as manifestações clínicas são mais subtis, como a hiperpigmentação do escroto e dos genitais ao nascimento, dificuldades alimentares e má evolução ponderal. O diagnóstico pode ser mais tardio, podendo instalar-se uma crise adisoniana. ${ }^{6-9,12}$

A forma CVS cursa com sinais de virilização pré-natal dos genitais externos no sexo feminino e por hipocortisolismo e pseudo-puberdade precoce em ambos os sexos. ${ }^{11,12}$ Pode manifestar-se por aceleração do crescimento e da maturação esquelética, crescimento do pénis nos rapazes e clitoromegalia nas meninas..$^{7,11,12}$

A forma mais ligeira é designada não clássica (NC) ou de início tardio. Não se manifesta por virilização genital neonatal, mas sim por sinais de hiperandrogenismo numa fase mais tardia. Na infância pode manifestar-se por aparecimento de pêlo púbico, acne e aceleração da idade óssea; na adolescência por acne, hirsutismo e irregularidades menstruais. No sexo masculino pode ser assintomática. ${ }^{11,12}$

A alteração bioquímica mais característica da deficiência de 21-OH é a elevação da 17-OHP, o principal substrato da enzima. Os valores basais da 17-OHP geralmente excedem $10000 \mathrm{ng} / \mathrm{dL}$, embora cerca de 10\% dos recém-nascidos possam apresentar níveis incialmente normais no período neonatal, especialmente se obtidos no primeiro dia de vida. Na forma não clássica, a 17-OHP basal pode ser normal ou ligeiramente elevada, mas mostra elevação significativa após prova de estimulação com a ACTH (1500 a $10000 \mathrm{ng} / \mathrm{dL}){ }^{12}$
Vários estudos analisaram a correlação entre o genótipo CYP21A2 e o fenótipo ${ }^{6,7,9,13}$ e concluíram que esta era elevada (80-90\%), embora não seja sempre possível prever o fenótipo com base no genótipo. . $^{8,13,14}$

Neste estudo pretende-se descrever o espectro de mutações do gene CYP21A2 em doentes com hiperplasia congénita da suprarrenal e avaliar a correlação genótipo-fenótipo numa coorte de doentes portugueses com deficiência de 21-hidroxílase, em idade pediátrica.

\section{MATERIAL E MÉTODOS:}

Estudo retrospetivo, que incluiu 22 crianças em idade pediátrica com critérios clínicos e laboratoriais de deficência de 21-OH, seguidas na Unidade de Endocrinologia do Centro Hospitalar do Porto entre 2000 e 2013.

O estudo molecular do gene CYP21A2 foi efetuado nos 22 doentes, a partir da extração de DNA de sangue periférico. Foram analisadas as mutações mais comuns e as deleções/conversões no gene do CYP21A2: P30L (exão 1), I2 splice (intrão 2), I172N (exão 4), V281L (exão 7), Q318X e R356W (exão 8), cluster E6 (exão 6), P453S (exão 10).

O estudo molecular não incluiu a sequenciação de todo o gene nos casos em que não foram encontradas mutações.

A pesquisa de grandes deleções/conversões no gene CYP21A2 foi realizada através da técnica de multiplex ligation-dependent probe amplification (MRC Holland).

A classificação fenotípica dos doentes foi determinada após revisão em conjunto pelos três Endocrinologistas Pediátricos da Unidade, baseada em critérios clínicos e hormonais, através da análise retrospetiva do registo clínico. Na presença de sinais clínicos e bioquímicos evidentes de crise adrenérgica (má evolução ponderal, hiponatrémia, hipercalémia e renina elevada) os doentes eram classificados na forma CPS. Neste grupo, o valor de 17-OHP no primeiro mês de vida era $>2500 \mathrm{ng} / \mathrm{dL}$. Os doentes do sexo feminino com genitais ambíguos, com 17-OHP elevada mas sem desequilíbrios hidroelectrolíticos ou nos quais foi detetada virilização precoce para além do período neonatal, foram classificadas como forma CVS.

Foram também incluídos neste grupo os rapazes que desenvolveram sinais e sintomas de hiperandrogenismo, aceleração do crescimento ou da idade óssea precocemente, 17-OHP elevada, mas sem evidência de perda de sal. A presença de sintomas de hiperandrogenismo em idade pré-púbere (aparecimento de pêlo púbico, acne, hirsustismo, odor corporal adulto e ausência de virilização completa dos genitais) e 17-OHP elevada foi utilizada para diagnosticar os doentes com forma NC. Estes doentes apresentavam valores de $17-\mathrm{OHP} \geq 500 \mathrm{ng} / \mathrm{dL}$.

\section{Categorização em grupos de mutações:}

As mutações responsáveis pela doença foram distribuídas em 4 grupos, dependendo do grau de atividade enzimática estimada para cada mutação, de acordo com os estudos in vitro publicados na literatura ${ }^{6,7}$. O grupo 0 , com atividade nula incluiu os doentes com mutações em ambos 


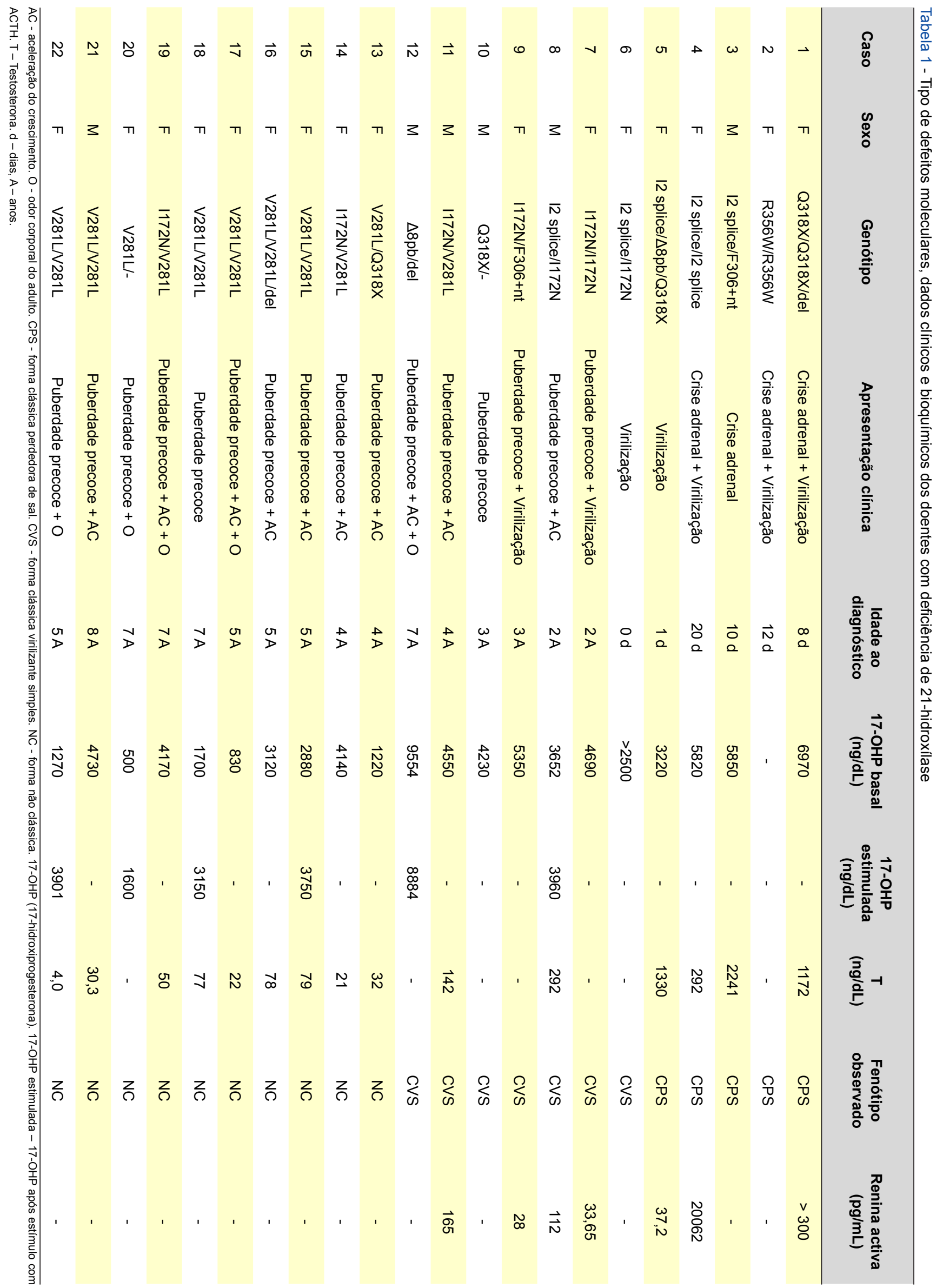


os alelos das quais resultam ausência de atividade enzimática (deleções/conversões de genes, $\Delta 8 \mathrm{bp}$, F306+t, cluster E6, Q318X, R356W). O grupo A incluiu os doentes homozigóticos para I2 splice ou heterozigóticos compostos para I2 splice e mutação anuladora, com atividade enzimática mínima (0-1\%). O grupo B incluiu os doentes com mutações I172N (com atividade enzimática residual de $\sim 2 \%$ ), em homozigotia ou heterozigóticos compostos com os grupos 0 , A ou B. O grupo C incluiu os genótipos compostos por uma mutação ligeira em pelo menos um dos alelos: V281L, P30L ou P453s ( 20-60\% de atividade enzimática residual, em homozigotia ou heterozigótico composto com os grupos 0 , A ou B. Um quinto grupo (sem classificação), incluiu doentes nos quais apenas se detetou uma mutação num dos alelos do CYP21A2.

O fenótipo esperado para os grupos 0 e A é CPS, para o grupo B CVS e grupo C NC.

Tendo em conta o genótipo dos doentes, estabeleceu-se o fenótipo previsível, de acordo com a atividade enzimática permitida pelo alelo menos afectado, e avaliou-se o grau de concordância entre o fenótipo esperado e o observado.

\section{RESULTADOS}

Foi efetuada genotipagem em 22 doentes não relacionados com diagnóstico de HCSR por deficiência de 21-hidroxílase. O tipo de defeitos moleculares, os dados clínicos e bioquímicos são apresentados na Tabela 1. Relativamente ao fenótipo clínico, 12 doentes $(54,5 \%)$ apresentaram a forma clássica da doença, 5 CPS (41,7\%) e 7 CVS $(58,3 \%)$, e 10 doentes $(45,5 \%)$ a forma NC.

Todos os doentes eram caucasianos e verificamos um claro predomínio do sexo feminino, 16/22 doentes.

A idade média ao diagnóstico da forma CPS foi 10,2 dias (mínimo 1, máximo 20 dias). Relativamente à forma CVS, apenas uma recém-nascida foi diagnosticada ao nascimento e, nos restantes, a idade média ao diagnóstico foi de 3,5 anos (mínimo 2 anos, máximo 7 anos). As formas não clássicas manifestaram-se em média aos 5,7 anos (mínimo 4 anos, máximo 8 anos).
Em dois casos (doentes 16 e 21), foi feito o diagnóstico posterior de deficiência de $21-\mathrm{OH}$ no irmão após rastreio familiar (não incluídos no estudo). Os restantes não apresentaram história familiar positiva.

Os valores de 17-OHP basal variaram entre os $2500 \mathrm{e}$ $9554 \mathrm{ng} / \mathrm{dL}$ nas formas clássicas e de 500 e $4730 \mathrm{ng} / \mathrm{dL}$ nas formas NC. Nos doentes que efetuaram prova com ACTH, o valor de 17-OHP aos 60 minutos variou entre 1600 e 8884 $\mathrm{ng} / \mathrm{dL}$.

A frequência dos defeitos moleculares encontrados na nossa amostra encontra-se discriminada na Tabela 2. Dos 22 doentes, foram identificadas mutações em ambos os alelos em 20 doentes $(90,9 \%)$, confirmando-se assim o diagnóstico genético da doença. Em 2 doentes apenas foi identificada uma mutação (Q318X e V281L). Na forma clássica, as mutações mais prevalentes foram o I2 splice (24\%) e I172N (24\%), seguindo-se a Q318X (16\%) e as deleções de genes (16\%). Na forma NC, a V281L foi a mutação mais frequente $(80 \%)$.

$\mathrm{Na}$ Tabela 3, encontram-se agrupadas o tipo de mutações de acordo com o grau de atividade enzimática esperada e o respetivo fenótipo clínico. Oito doentes apresentavam mutações em homozigotia, 12 em heterozigotia composta e 2 em heterozigotia para uma mutação. Verificamos uma concordância genótipo-fenótipo de $81,8 \%$ (18/22 doentes), tendo sido total nos grupos $A$ e $B$, nos quais era esperada apresentação CPS e CVS respetivamentente. Uma recém-nascida diagnosticada no primeiro dia de vida por ambiguidade genital, cujo fenótipo esperado era CPS, apesar de não ter evidenciado sinais de perda de sal foi classificada como tal, já que foi necessário tratamento com mineralocorticóide e suplementos de sal. Neste caso, o diagnóstico e tratamento foram precoces, tendo-se assim evitado a crise adrenérgica. No grupo 0 , em que era esperado fenótipo CPS, um de três casos foi discordante (concordância genótipo-fenótipo de 66,7\%). Tratava-se de um rapaz com mutação compatível com forma CPS e manifestações clínicas de forma CVS. O diagnóstico foi feito aos 7 anos, por aparecimento de sinais pubertários e aceleração do crescimento, com valores de 17-OHP basal ao diagnós-

Tabela 2 - Frequência de mutações em 45 alelos afectados de 22 pacientes não relacionados com deficiência de 21-hidroxílase

\begin{tabular}{ccccccc}
\hline Mutação & \multicolumn{3}{c}{ Número de alelos } & \multicolumn{3}{c}{$\%$ Total } \\
& Clássica & NC & Total & Clássica & NC & Total \\
\hline R356W & 2 & 0 & 2 & $8 \%$ & 0 & $4,4 \%$ \\
Q318X & 4 & 1 & 5 & $16 \%$ & $5 \%$ & $11,1 \%$ \\
Deleções & 4 & 1 & 5 & $16 \%$ & $5 \%$ & $11,1 \%$ \\
I2 splice & 6 & 0 & 6 & $24 \%$ & 0 & $13,3 \%$ \\
F306+nt & 2 & 0 & 2 & $8 \%$ & 0 & $4,4 \%$ \\
I172N & 6 & 2 & 8 & $24 \%$ & $10 \%$ & $17,8 \%$ \\
V281L & 1 & 16 & 17 & $4 \%$ & $80 \%$ & $37,8 \%$ \\
Total & 25 & 20 & 45 & $100 \%$ & $100 \%$ & $100 \%$ \\
\hline
\end{tabular}


Tabela 3 - Genótipos agrupados de acordo com a gravidade esperada das mutações envolvidas e correlação com o respetivo fenótipo

\begin{tabular}{|c|c|c|c|c|c|c|c|}
\hline \multirow{2}{*}{ Grupo } & \multirow{2}{*}{ Genótipo } & \multirow{2}{*}{$\begin{array}{c}\mathrm{N}^{\circ} \text { de } \\
\text { doentes }\end{array}$} & \multicolumn{3}{|c|}{ Fenótipo observado } & \multirow{2}{*}{$\begin{array}{l}\text { Fenótipo } \\
\text { esperado }\end{array}$} & \multirow{2}{*}{$\begin{array}{c}\text { Correlação } \\
\text { Genótipo-fenótipo }\end{array}$} \\
\hline & & & CPS & CVS & NC & & \\
\hline \multirow[t]{3}{*}{ Grupo 0} & R356W/R356W & 1 & 1 & & & CPS & $100 \%$ \\
\hline & Q318X/Q318X/del & 1 & 1 & & & CPS & $100 \%$ \\
\hline & $\Delta 8 \mathrm{pb} / \mathrm{del}$ & 1 & & 1 & & CPS & $0 \%$ \\
\hline \multirow[t]{3}{*}{ Grupo A } & I2 splice/F306+nt & 1 & 1 & & & CPS & $100 \%$ \\
\hline & I2 splice/ $\Delta 8 p b / Q 318 X$ & 1 & 1 & & & CPS & $100 \%$ \\
\hline & I2 splice//2 splice & 1 & 1 & & & CPS & $100 \%$ \\
\hline \multirow[t]{3}{*}{ Grupo B } & I2 splice//172N & 2 & & 2 & & CVS & $100 \%$ \\
\hline & I172N/I172N & 1 & & 1 & & CVS & $100 \%$ \\
\hline & I172N/F306+nt & 1 & & 1 & & CVS & $100 \%$ \\
\hline \multirow[t]{4}{*}{ Grupo C } & I172N/V281L & 3 & & 1 & 2 & NC & $66,7 \%$ \\
\hline & V281L/V281L & 5 & & & 5 & $\mathrm{NC}$ & $100 \%$ \\
\hline & V281L/V281L/del & 1 & & & 1 & NC & $100 \%$ \\
\hline & V281L/Q318X & 1 & & & 1 & NC & $100 \%$ \\
\hline \multirow[t]{2}{*}{ Não clasificado } & Q318X/- & 1 & & 1 & & Asintomático & $0 \%$ \\
\hline & V281L/- & 1 & & & 1 & Asintomático & $0 \%$ \\
\hline Total & & 22 & 5 & 7 & 10 & & $81,8 \%$ \\
\hline
\end{tabular}

tico de $9554 \mathrm{ng} / \mathrm{dL}$ e $17-O H P$ estimulada de $8884 \mathrm{ng} / \mathrm{dL}$

No grupo C a concordância foi de $90 \%$ (9/10 doentes).

O caso discordante refere-se a um rapaz, com genótipo compatível com forma NC e que apresentava fenótipo clínico CVS. Aos 4 anos manifestava sinais de pseudo-puberdade precoce e aceleração marcada do crescimento, com valor de testosterona de $142 \mathrm{ng} / \mathrm{dL}$ e 17-OHP basal de $4550 \mathrm{ng} / \mathrm{dL}$.

Dois doentes, um do sexo masculino com manifestações clínicas de forma CVS aos 3 anos e outro do sexo feminino com fenótipo NC diagnosticada aos 7 anos, apresentavam apenas uma mutação, pelo que seria de esperar que fossem assintomáticos.

\section{DISCUSSÃO}

Neste estudo, descrevemos o espectro mutacional, a frequência alélica e a correlação genótipo-fenótipo numa amostra de 22 doentes portugueses não relacionados com HCSR por deficiência de 21-OH.

Todos os doentes apresentaram as mutações descritas na literatura ${ }^{6,7,11}$, geradas pela recombinação entre o gene ativo CYP21 e o respetivo pseudogene.

O espectro mutacional encontrado na nossa amostra foi, em geral, semelhante ao encontrado noutros estudos. ${ }^{6,715-18}$ As mutações mais prevalentes na forma clássica foram I2 splice (24\%) e I172N (24\%), seguindo-se a Q318X (16\%) e as deleções de genes (16\%). A frequência da mutação Q318X foi maior na nossa amostra do que na maioria das populações, embora concordante com outros estudos. ${ }^{19,20} \mathrm{Na}$ forma NC, a V281L foi a mutação preponderante $(80 \%)$, como em todas as populações estudas, ${ }^{6-9,17,18,20-23}$ tal como já descrito num estudo prévio da população portuguesa. ${ }^{13}$ Verificamos uma boa correlação genótipo-fenótipo (correlação global de $81,8 \%$ ) e o genótipo permitiu prever o fenótipo em $83,3 \%, 100 \%$ e $90 \%$ dos doentes com mutações compatíveis com a forma CPS, CVS e NC, respetivamente. Os grupos A e B foram aqueles em que obtivemos maior concordância. Esta diferença em relação a outros estudos ${ }^{14,16-21,24}$ (em que a maior concordância ocorreu nos grupos extremos de gravidade) poderá explicar-se pelo reduzido tamanho da amostra e pelo tipo de mutações encontradas. Num dos doentes (caso 12), o fenótipo observado foi menos grave do que o esperado. Tratava-se de um doente com 2 mutações anuladoras, cuja forma de apresentação foi CVS. Nestes casos, alguns estudos $^{14,25}$ colocam a hipótese de que as variações genéticas no CYP2C19 y CYP3A4, a presença de outras enzimas com capacidade de modular o equilíbrio hidroelectrolítico dos doentes com HCSR, ou uma combinação de fatores desconhecidos possam modelar a ação esteróide e, deste modo, explicar estes achados.

Pelo contrário, um dos doentes (caso 11) manifestou uma forma mais grave do que a prevista pelo genótipo. Apresentava manifestações clínicas compatíveis com forma CVS, com genótipo preditor de forma NC. A grande variabilidade da atividade enzimática da mutação I172N ${ }^{14}$ poderá contribuir para a variabilidade fenotípica e ser uma possível explicação neste caso. As discrepâncias observadas podem também ser explicadas por novas mutações que não foram pesquisadas à data do diagnóstico (e que poderão justificar o fenótipo das 2 crianças em que apenas foi detectada uma mutação), genotipagem incompleta, heterozigotia composta para duas ou mais mutações e outras variações genéticas na biossíntese ou na sensibilidade aos androgénios.

Neste estudo verificamos que mais de metade dos doentes apresentaram a forma clássica da doença $(54,5 \%)$, o que não seria de esperar. ${ }^{25}$ Este achado pode refletir dificuldades no diagnóstico, bem como a subvalorização das formas com apresentação clínica mais ligeira. ${ }^{1,23}$ No 
entanto, não podemos excluir que estes resultados resultem da reduzida dimensão da amostra. Por outro lado, a forma não clássica cursa com uma variedade de sintomas de hiperandrogenismo, o que coloca dificuldades no diagnóstico diferencial, nomeadamente com Síndrome do Ovário Poliquístico. Importa salientar que a nossa amostra apenas incluiu doentes até aos 18 anos de idade e que muitas formas não clássicas apenas são diagnosticadas na idade adulta por infertilidade. ${ }^{26}$

Foi também evidente o predomínio do sexo feminino, o que está de acordo com a literatura. Como se trata de uma patologia autossómica recessiva, ocorre igualmente nos dois sexos. ${ }^{23} \mathrm{~A}$ assimetria na distribuição do género observada, principalmente na forma NC, em que todas as crianças apresentaram genótipo V281L em pelo menos um dos alelos (com uma proporção de 9 raparigas para apenas 1 rapaz) faz-nos pensar que os rapazes possam ser mais vezes subdiagnosticados, pelo facto de os sinais de hiperandrogenismo serem menos óbvios, tal como descrito previamente. ${ }^{17}$

\section{CONCLUSÕES}

O conhecimento da especificidade étnica das mutações do CYP21A2 é importante em todas as formas de hiperplasia congénita da suprarrenal. $O$ facto de existir um número

\section{REFERÊNCIAS}

1. Merke DP, Bornstein SR. Congenital adrenal hyperplasia. Lancet. 2005;365:2125-36.

2. Miller WL. Molecular biology of steroid hormone synthesis. Endocr Rev. 1988;9:295-318.

3. White PC, Tusie-Luna MT, New MI, Speiser PW. Mutations in steroid 21-hydroxylase (CYP21). Hum Mutat. 1994;3:373-8.

4. Miller WL. Genetics, diagnosis and management of 21-hydroxylase deficiency. J Clin Endocrinol Metab. 1994;78:241-6.

5. Koppens PF, Hoogenboezem T, Degenhart HJ. Carriership of a defective tenascin- $X$ gene in steroid 21-hydroxylase deficiency patients: TNXB-TNXA hybrids in apparent large-scale gene conversions. Hum Mol Genet. 2002;11:2581-90.

6. Speiser PW, Dupont J, Zhu D, Serrat J, Buegeleisen M, Tusie-Luna $\mathrm{MT}$, et al. Disease expression and molecular genotype in congenital adrenal hyperplasia due to 21-hydroxylase deficiency. J Clin Invest. 1992;90:584-95.

7. Wedell A, Thilen A, Ritzen EM, Stengler B, Luthman H. Mutational spectrum of the steroid 21-hydroxylase gene in Sweden: implications for genetic diagnosis and association with disease manifestation. J Clin Endocrinol Metab. 1994;78:1145-52.

8. Wilson RC, Mercado AB, Cheng KC, New MI. Steroid 21-hydroxylase deficiency: genotype may not predict phenotype. J Clin Endocrinol Metab. 1995;80:2322-9.

9. Ezquieta B, Oliver A, Gracia R, Gancedo PG. Analysis of steroid 21-hydroxylase gene mutations in the Spanish population. Hum Genet. 1995;96:198-204

10. Pang SY, Wallace MA, Hofman L, Thuline HC, Dorche C, Lyon IC, et at. Worldwide experience in newborn screening for classical congenital adrenal hyperplasia due to 21-hydroxylase deficiency. Pediatrics. 1998;81:866-74.

11. Speiser PW, Dupont B, Rubinstein P, Piazza A, Kastelan A, New MI. High frequency of non-classical steroid 21-hydroxylase deficiency. Am J Hum Genet. 1985;37:650-67.

12. White PC, Speiser PW. Congenital adrenal hyperplasia due to 21-hydroxylase deficiency. Endocr Rev. 2000;21:245-91.

13. Friães A, Rêgo AT, Aragüés JM, Moura LF, Mirante A, Mascarenhas MR, et al. CYP21A2 mutations in Portuguese patients with congenital adrenal hyperplasia: identification of two novel mutations and characterization of limitado de mutações responsáveis pela maioria dos casos de HCSR, já descrita na literatura e corroborada no nosso estudo, torna a deteção de mutações útil para o rastreio pré-natal. O diagnóstico pré-natal com análise do DNA obtido por amniocentese ou por biópsia das vilosidades coriónicas tem implicações terapêuticas importantes no sexo feminino, podendo evitar a virilização dos genitais externos e a atribuição incorrecta do sexo. Por outro lado, pode antecipar a instalação de uma crise adrenérgica potencialmente fatal e evitar o hiperandrogenismo durante a infância.

Apesar de alguma variabilidade na expressão clínica, verificamos na maioria dos casos uma boa correlação do genótipo CYP21A2 e o fenótipo de HCSR. Assim, o nosso estudo reforça a importância do estudo molecular do CYP21A2 como ferramenta complementar na predição da gravidade da doença e no aconselhamento genético e pré-natal.

\section{CONFLITOS DE INTERESSE}

Os autores declararam não existirem quaisquer conflitos de interesse relativamente a este trabalho.

\section{FONTES DE FINANCIAMENTO}

Os autores declararam que o presente trabalho não foi financiado. four different partial gene conversions. Mol Genet Metab. 2006;88:5865

14. Finkielstain GP, Chen W, Mehta SP, Fujimura FK, Hanna RM, Van Ryzin C, et al. Comprehensive genetic analysis of 182 unrelated families with congenital adrenal hyperplasia due to 21-hydroxylase deficiency. J Clin Endocrinol Metab. 2011;96:E161-72.

15. Wedell A. Molecular genetics of congenital adrenal hyperplasia (21hydroxylase deficiency): implications for diagnosis, prognosis and treatment. Acta Paediatr. 1998;87:159-64.

16. Krone N, Braun A, Roscher AA, Knorr D, Schwarz HP. Predicting phenotype in steroid 21-hydroxylase deficiency? Comprehensive genotyping in 155 unrelated, well defined patients from southern Germany. J Clin Endocrinol Metab. 2000;85:1059-65.

17. New MI, Abraham M, Gonzalez B, Dumic M, Razzaghy-Azar M, Chitayat $\mathrm{D}$, et al. Genotype-phenotype correlation in 1,507 families with congenital adrenal hyperplasia owing to 21-hydroxylase deficiency. Proc Natl Acad Sci U S A. 2013;110:2611-6.

18. Vieira A, Paiva S, Baptista C, Ruas L, Silva J, Gonçalves J, et al. Late onset congenital adrenal hyperplasia due to 21-hydroxylase deficiency: revision of literature and preconception genetic study of five couples. Acta Med Port. 2011;24:99-110.

19. Dracopoulou-Vabouli M, Maniati-Christidi M, Dacou-Voutetakis C. The spectrum of molecular defects of the CYP21 gene in the Hellenic population: Variable concordance between genotype and phenotype in the different forms of congenital adrenal hyperplasia. J Clin Endocrinol Metab. 2001;86:2845-8.

20. Carrera P, Bordone L, Azzani T, Brunelli V, Garancini MP, Chiumello $\mathrm{G}$, et al. Point mutations in Italian patients with classic, non-classic, and cryptic forms of steroid 21-hydroxylase deficiency. Hum Genet. 1996;98:662-5.

21. Bachega TA, Billerbeck AE, Madureira G, Marcondes JA, Longui CA, Leite MV, et al. Molecular genotyping in Brazilian patients with the classical and nonclassical forms of 21-hydroxylase deficiency. J Clin Endocrinol Metab. 1998;83:4416-9.

22. Rumsby G, Avey CJ, Conway GS, Honour JW. Genotype-phenotype analysis in late onset 21-hydroxylase deficiency in comparison to the classical forms. Clin Endocrinol. 1998;48:707-11.

23. New MI. Extensive clinical experience: nonclassical 21-hydroxylase 
deficiency. J Clin Endocrinol Metab. 2006:91:4205-14.

24. Skordis N, Kyriakou A, Tardy V, loannou YS, Varvaresou A, Dracopoulou-Vabouli M, et al. Molecular defects of the CYP21A2 gene in Greek-Cypriot patients with congenital adrenal hyperplasia. Horm Res Paediatr. 2011;75:180-6.

25. Gomes LG, Huang N, Agrawal V, Mendonça BB, Bachega TA, Miller
WL. Extraadrenal 21-hydroxylation by CYP2C19 and CYP3A4: effect on 21-hydroxylase deficiency. J Clin Endocrinol Metab. 2009;94:89-95. 26. Marques CJ, Pignatelli D, Carvalho B, Barceló J, Almeida AC, Fernandes $S$, et al. Mutational characterization of steroid 21-hydroxylase gene in Portuguese patients with congenital adrenal hyperplasia. Exp Clin Endocrinol Diabetes. 2010;118:505-12. 


\section{Hiperplasia Congénita da Suprarrenal por Deficiência de 21-Hidroxílase: Correlação Genótipo-Fenótipo}

Acta Med Port 2015:28:56-62

Publicado pela Acta Médica Portuguesa, a Revista Científica da Ordem dos Médicos

Av. Almirante Gago Coutinho, 151

1749-084 Lisboa, Portugal.

Tel: +351218428215

E-mail: submissao@actamedicaportuguesa.com

www.actamedicaportuguesa.com

ISSN:0870-399X | e-ISSN: 1646-0758

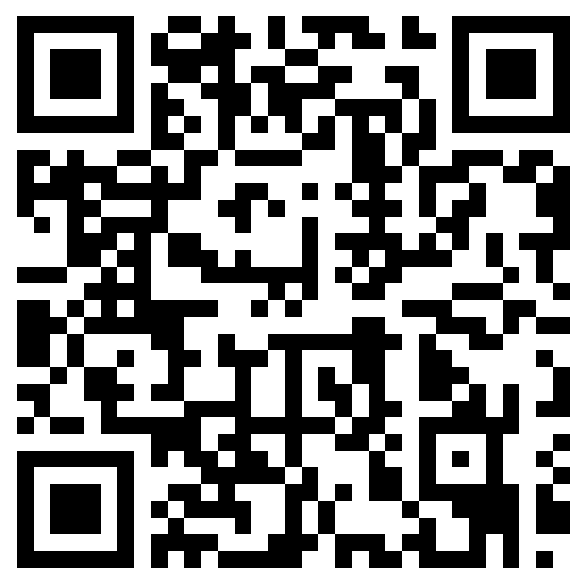

